\title{
Advanced Molecular Imaging for Exploring Classic Conditioning and Extinction
}

\author{
Songji Zhao ${ }^{1}$, Chowdhury Nusrat Fatema ${ }^{2}$, Jingmin Zhao $^{3}$, and Guangxian $\mathrm{Nan}^{3}$ \\ ${ }^{1}$ Advanced Clinical Research Center, Fukushima Global Medical Science Center, Fukushima Medical University, Fukushima, Japan; \\ ${ }^{2}$ Department of Oral Diagnosis and Medicine, Hokkaido University, Sapporo, Japan; and ${ }^{3}$ Department of Neurology, China-Japan \\ Union Hospital of Jilin University, Changchun, China
}

$\mathbf{N}$ early 100 y ago, Ivan Pavlov presented the most famous example of classic conditioning, which occurs when a conditioned stimulus is paired with an unconditioned stimulus. Usually, the conditioned stimulus is a neutral (e.g., the sound of a tuning fork) and the unconditioned stimulus is biologically potent (e.g., food) and produces an unconditioned response (e.g., salivation). In anxiety disorders, such as posttraumatic stress disorder (PTSD), a trauma can be considered an unconditioned stimulus; this type of conditioned fear fails to extinguish, and reminders of traumatic events can cause pathologic conditioned fear responses for decades after danger has passed (1). PTSD affects up to $40 \%$ of individuals with over-lifetime exposure to traumatic events $(2,3)$. Over the past decade, there has been a surge of interest in the experimental extinction of fear for its own sake. Beyond the interest in the basic mechanisms of learning and memory, this renewed interest in fear extinction is due in large part to the clinical significance of this extinction for the treatment of a variety of psychiatric

\section{See page 1474}

disorders (4). Although exposure-based therapy (i.e., conceptually based on fear extinction) has been widely used in the treatment of PTSD (2), its underlying mechanism remains unclear. Recently, ${ }^{18} \mathrm{~F}$ FDG PET has been increasingly applied to the characterization of brain regional metabolic activation, and methods of whole-brain imaging analysis such as statistical parametric mapping have been applied more extensively to determine the relationship of measured behavior with brain metabolic changes in humans and rodents (5-8).

In the current issue of The Journal of Nuclear Medicine, Zhu et al. (9) reported their novel finding of neurofunctional changes in a PTSD rat model. They investigated the glucose metabolic changes before and after exposure-based therapy by ${ }^{18}$ F-FDG PET imaging analysis. They found an enhanced glucose metabolism in the bilateral amygdale after fear conditioning and the right posterior insular cortex during fear extinction retrieval. The number of c-Fos (a neuronal activation marker)-positive cells in the posterior insular cortex was also signif-

\footnotetext{
Received Apr. 3, 2016; revision accepted Apr. 5, 2016.

For correspondence or reprints contact: Songji Zhao, Advanced Clinical Research Center, Fukushima Global Medical Science Center, Fukushima Medical University, 1 Hikarigaoka, Fukushima 960-1295, Japan.

E-mail: zhao-s@fmu.ac.jp

Published online May 19, 2016.

COPYRIGHT (C) 2016 by the Society of Nuclear Medicine and Molecular Imaging, Inc.

DOI: $10.2967 /$ jnumed.116.174383
}

icantly increased after the fear conditioning and positively correlated with ${ }^{18} \mathrm{~F}-\mathrm{FDG}$ accumulation. They concluded that the amygdala plays a key role in fear memory formation and that, most importantly, the insular cortex is related to the retrieval of extinction memory.

In the brain, glucose metabolism provides approximately $95 \%$ of adenosine triphosphate required for brain function. Under normal physiologic conditions, glucose metabolism is tightly connected to neuronal activity, because changes in neuronal activity induced by a disease are directly reflected in an alteration of glucose metabolism. Therefore, ${ }^{18} \mathrm{~F}$-FDG PET is currently the most accurate in vivo method for the clinical investigation of regional human brain metabolism in health and disease states. The current study by Zhu et al. (9) presented a systemwide analysis of glucose metabolism during behavioral training and provided a useful translational tool for future clinical applications to the treatment of psychiatric disorders.

For the first time, this study provided evidence for neurofunctional changes by direct comparison of neuronal activity in rats before and after classic conditioning and extinction. The involvement of the amygdala and insular cortex indicated that these 2 regions are associated with PTSD. Future investigations using PET combined with functional MRI, electrophysiologic recording, and optogenetics could further our understanding of targeted therapy and provide its conceptual basis.

\section{DISCLOSURE}

No potential conflict of interest relevant to this article was reported.

\section{REFERENCES}

1. VanElzakker MB, Dahlgren MK, Davis FC, Dubois S, Shin LM. From Pavlov to PTSD: the extinction of conditioned fear in rodents, humans, and anxiety disorders. Neurobiol Learn Mem. 2014;113:3-18.

2. Powers MB, Halpern JM, Ferenschak MP, Gillihan SJ, Foa EB. A meta-analytic review of prolonged exposure for posttraumatic stress disorder. Clin Psychol Rev. 2010;30:635-641.

3. Ressler KJ, Mercer KB, Bradley B, et al. Post-traumatic stress disorder is associated with PACAP and the PAC1 receptor. Nature. 2011;470:492-497.

4. Dunsmoor JE, Niv Y, Daw N, Phelps EA. Rethinking extinction. Neuron. 2015;88:47-63.

5. Jones T, Rabiner EA. PET Research Advisory Company. The development, past achievements, and future directions of brain PET. J Cereb Blood Flow Metab. 2012;32:1426-1454

6. Small GW, Bookheimer SY, Thompson PM, et al. Current and future uses of neuroimaging for cognitively impaired patients. Lancet Neurol. 2008;7:161-172.

7. Miyamoto M, Kuroda S, Zhao S, et al. Bone marrow stromal cell transplantation enhances recovery of local glucose metabolism after cerebral infarction in rats: a serial ${ }^{18}$ F-FDG PET study. $J$ Nucl Med. 2013;54:145-150.

8. Endepols H, Sommer S, Backes H, Wiedermann D, Graf R, Hauber W. Effortbased decision making in the rat: an $\left[{ }^{18} \mathrm{~F}\right]$ fluorodeoxyglucose micro positron emission tomography study. J Neurosci. 2010;30:9708-9714.

9. Zhu Y, Du R, Zhu Y, et al. PET mapping of neurofunctional changes in a posttraumatic stress disorder model. J Nucl Med. 2016;57:1474-1477. 